\title{
A Condition-Based Maintenance Strategy for Heterogeneous Populations
}

\author{
Mimi Zhang*1, Zhisheng $\mathrm{Ye}^{2}$, and Min Xie ${ }^{1}$ \\ ${ }^{1}$ Department of Systems Engineering and Engineering Management, City University of Hong Kong \\ ${ }^{2}$ Department of Applied Mathematics, The Hong Kong Polytechnic University
}

\begin{abstract}
This paper develops a maintenance strategy, called inspection-replacement policy, to cope with heterogeneous populations. Burn-in is the procedure by which most of the defective products in a heterogeneous population can be identified and removed prior to being placed in service. However, modern manufacturing is so well developed that a defective product is able to function for a long period of time even under aggravated operational conditions. Instead of weeding defective products out via costly burn-in tests, use can be made of them in field operation where maintaining actions will be performed to prevent early in-use failures. The inspection-replacement policy consists of an inspection, conducted in an early stage with the purpose of identifying and replacing defective products, and a preventive replacement, carried out at a later stage to prevent wear-out failures. The preventive-replacement time is dynamically determined, depending on the information obtained by the inspection. The inspection-replacement policy is compared with a joint burn-in and age-based-replacement policy to show its practicability and competence.
\end{abstract}

Keywords: Burn-in test; gamma process; mixture distribution; preventive replacement; Wiener process.

\section{INTRODUCTION}

To maintain competitive advantage, manufacturers endeavor as much as possible to produce reliable products. However, during the manufacturing process, some unavoidable manufacturing defects could be introduced, e.g. defects in the raw material, leading to a heterogeneous population of products. The heterogeneous population contains a small proportion of weak/defective products. Compared with the normal products, the weak products have a shorter mean lifetime and are prone to giving rise to early in-use failures. The early in-use failures will cause substantial costs and sometimes are hazardous. In fact, it is not uncommon to observe a heterogeneous population with two sub-populations: a weak sub-population and a normal sub-population. For example, it is widely believed that integrated circuits consist of a small proportion of weak

\footnotetext{
${ }^{*}$ Corresponding author. Tel.: +852-5446-5352. E-mail: mmzhang5-c@my . cityu. edu. hk.
} 
items which have much shorter mean lifetime. The GaAs laser data set, provided by Meeker and Escobar (1998), is a typical sample consisting of a sub-group of normal devices and a subgroup of weak devices; see Tsai et al. (2011). Scarf and Cavalcante (2012) considered component heterogeneity when developing an age-based maintenance policy for a single-component system; they assumed that the population of components comprises a mixture of the weak and the strong. Berrade et al. (2013) developed a maintenance policy involving periodic inspections, in which inspections are subject to error; they assumed that the time a system spends in the defective state is a random variable from a mixture distribution. Recent research on heterogeneous data can be found in Cha and Finkelstein (2013), Eriolu et al. (2013) and Kazmi et al. (2013), among others.

A common practice to tackle a heterogeneous population is to screen out the weak products by means of a burn-in test. Burn-in is an engineering procedure implemented at the end of the manufacturing process. In a burn-in test, all the products are subjected to harsh electrical and thermal conditions that emulate the field operational conditions. At the end of the burn-in test, only the functioning products will be shipped to customers. There has been a bulk of research on developing economical burn-in tests; see Cha and Finkelstein (2010), Pearn et al. (2013), Post and Bhattacharyya (2012) and Yuan and Kuo (2010), among others. With the rapid development of modern manufacturing technology, even a weak product is able to operate for a fairly long period of time under aggravated operational conditions. Weeding out weak products via traditional age-based burn-in approach is therefore ineffectual. When there is a performance characteristic whose evolution is closely associated with the lifetime of the product, the condition-based burn-in approach is an attractive alternative (Xiang et al., 2013, Ye et al. 2012). Because a weak product most often deteriorates faster than a normal product, a condition-based burn-in test exercises all the products for a certain period of time and scraps the products with deterioration levels higher than a pre-specified cut-off level. After the burn-in test, all the products with deterioration levels lower than the cut-off level are shipped to customers. In field operation, preventive repairs are often scheduled to further improve reliability and reduce operational costs. See Ahmad and Kamaruddin (2012) for an overview of two maintenance techniques widely adopted by industrial engineers: time-based maintenance and condition-based maintenance. Nonetheless, it is well known that burn-in is costly, including such as burn-in set-up cost, burn-in operational cost and repair/scrap cost from a burn-in failure. Scilicet, by adopting the burn-in procedure, we make a trade-off between early in-use failures and a reduced yield due to the burn-in costs. It is worth noting that nowadays even a weak product is able to operate for a long term. A more judgmatic approach to tackling a heterogeneous population is to directly put all the products into field operation and replace the weak products before they fail. By virtue of appropriately scheduled inspections, early in-use failures can be mitigated, and the failure cost can be countervailed by the long-time operating income. Even if a burn-in test is able to identify most of the defective products, it will cause damage to the normal products, shortening the mean lifetime of the normal products (Cha and Finkelstein, 2011; Ye et al., 2011).

To make full use of weak products and avoid impairing normal products, we develop the inspection-replacement policy for heterogeneous populations with the assumption that a suitable performance characteristic of the products is available. The inspection-replacement policy directly puts all the products in a heterogeneous population into field use without burn-in. The physical degradation of each product will be measured at time $b(>0)$. If the degradation of a product exceeds a critical threshold, then it will be treated like a weak product and replaced 
at time $b$. If the degradation does not exceed the critical threshold, then it will be treated like a normal product and will be preventively replaced at time $R(>b)$, if it survives to time $R$. A schematic diagram of the inspection-replacement policy is shown in Figure 1 . The purpose of

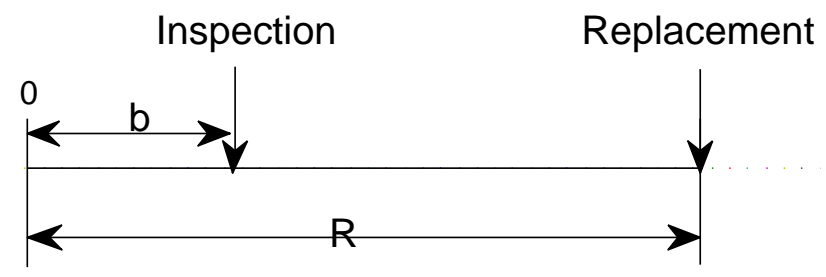

Figure 1: Schematic of the inspection-replacement policy.

the inspection is to identify and replace weak products, whereas the preventive replacement is to replace aged normal products. If the inspection and the critical threshold are well determined, most of the weak products will be identified by the inspection. It is not difficult to see that the role of the inspection is analogous to a burn-in test except that the inspection is carried out in the field operation. However, there will be some figures of merit by replacing the burn-in approach with the inspection-replacement policy. First of all, the inspection-replacement policy does not need to burn-in products, and therefore saves burn-in costs and avoids impairing normal products. Secondly, the inspection-replacement policy makes full use of weak products, and therefore is expected to yield more profits. Last but not least, the preventive-replacement time $R$ for each product is dynamically determined, depending on the physical degradation of the product obtained by the inspection. The on-line updating technique is expected to return more cost-efficient maintenance policy.

The rest of the paper is organized as follows. Section 2 details the inspection-replacement policy. The long-run cost rate function is derived. A joint burn-in and age-based-replacement policy serving as a benchmark is introduced. Section 3 draws the inspection-replacement policy on two predominant stochastic processes: the gamma process and the Wiener process. Section 4 presents numerical examples to elaborate the methodology developed in Section 2 and Section 3. The GaAs laser data set is analyzed. Contradistinctive analysis and sensitivity analysis are performed by using numerical simulations. Section 5 concludes the paper.

\section{MAINTENANCE STRATEGY \& COST RATE FUNCTION}

The product concerned stands for a component which is randomly drawn from a heterogeneous population. When put into operation, the component undergoes deterioration with the degradation process denoted by $\left\{X_{t}, t \geq 0\right\}$, assuming $X_{0}=0$ as with the convention. A component is considered to have failed if its deterioration level reaches a given failure threshold, denoted by $l(>0)$, which is a known constant. Define $T$ to be a random variable representing the first hitting time of the degradation process $\left\{X_{t}, t \geq 0\right\}$ to the failure threshold $l$. The inspectionreplacement policy is detailed as follows. After the installation at time 0 , the component is subject to inspection at a pre-determined epoch $b(>0)$. The component will be treated like a weak component and will be replaced by a new component if it survives beyond the point, i.e. $T>b$, yet with the deterioration level $X_{b}$ equating or exceeding a critical threshold $\vartheta(<l)$. The new backup component is randomly drawn from the same heterogeneous population. In the case that $T>b$ and $X_{b}<\vartheta$, a preventive-replacement time $R(>b)$ will be scheduled. $R$ is 
the time point upon which the reliability of the component drops to a pre-determined reliability threshold $\delta(0<\delta<1)$. Note that the preventive-replacement time $R$ is on-line calculated, depending on the deterioration level $X_{b}$. In the case that the component survives beyond the preventive-replacement time, i.e. $X_{b}<\vartheta$ and $T>R$, it will be preventively replaced at time $R$ by a new component. In the event that the component fails unexpectedly, i.e. $0<T<b$ or $b<T \leq R$, it will be immediately replaced by a new component. The intention of the inspection is to screen out poor-quality components and hence to prevent early failures. The purpose of the preventive replacement is to reduce wear-out failures caused by normal components. Other mild assumptions are given as follows.

- Starting from the installation of a device, the wear trajectory is taken to have an upward trend though not necessarily monotonically increasing.

- Inspection is perfect in the sense that it reveals the true degradation level of a device and does not change the condition of the device.

- Replacement time is negligible compared to the expected lifetime of the devices. Failure is self-announcing and can be observed instantaneously.

Components with $X_{b} \geq \vartheta$ are replaced at the condition-monitoring point instantaneously, and the average cost of replacing a component is $C_{r}$. Preventive replacement of a component at time $R$ is instantaneous and again costs $C_{r}$. The cost of each inspection is $C_{I}$, and the cost of a failure is $C_{f}$. Practical conditions define the constraints on the costs as follows: $C_{I}<C_{r}<C_{f}$. The decision variables of the inspection-replacement policy are the condition-monitoring epoch, $b$, and the corresponding critical threshold, $\vartheta$. The reliability threshold, $\delta$, is pre-determined by domain experts. A conservative engineer may set a high value of reliability threshold.

Let random variable $V$ denote the length of a single replacement cycle, i.e. the time from the installation of a component to its replacement. In every replacement cycle, one of the following 4 exclusive events occurs.

$\mathbf{E}_{1}=\{0<T \leq b\}$ : A component fails before or at the condition-monitoring epoch $b$, costing $C_{f}+C_{r}$.

$\mathbf{E}_{2}=\left\{T>b, X_{b} \geq \vartheta\right\}:$ A component survives to $b$ and is replaced at $b$, costing $C_{I}+C_{r}$.

$\mathbf{E}_{3}=\left\{X_{b}<\vartheta, b<T \leq R\right\}$ : A component, with $X_{b}<\vartheta$, fails at or before the preventivereplacement time $R$, costing $C_{I}+C_{f}+C_{r}$.

$\mathbf{E}_{4}=\left\{X_{b}<\vartheta, T>R\right\}:$ A component, with $X_{b}<\vartheta$, survives to the preventive-replacement time $R$ and is replaced at $R$, costing $C_{I}+C_{r}$.

After each replacement, the process renews. The total cost incurred in a replacement cycle can be formulated to be

$$
\begin{aligned}
C & =\left(C_{f}+C_{r}\right) I\left(\mathrm{E}_{1}\right)+\left(C_{I}+C_{r}\right) I\left(\mathrm{E}_{2}\right)+\left(C_{I}+C_{f}+C_{r}\right) I\left(\mathrm{E}_{3}\right)+\left(C_{I}+C_{r}\right) I\left(\mathrm{E}_{4}\right) \\
& =C_{r}+C_{f} I\left(\mathrm{E}_{1}\right)+C_{I} I\left(\mathrm{E}_{2}\right)+\left(C_{I}+C_{f}\right) I\left(\mathrm{E}_{3}\right)+C_{I} I\left(\mathrm{E}_{4}\right) .
\end{aligned}
$$

$I(\cdot)$ is the indicator function which equals 1 if the argument is true and 0 otherwise. Hence, the expected total cost incurred in a replacement cycle is

$$
\begin{aligned}
E[C] & =C_{r}+C_{f} p_{1}+C_{I} p_{2}+\left(C_{I}+C_{f}\right) p_{3}+C_{I} p_{4} \\
& =C_{r}+C_{I}+\left(C_{f}-C_{I}\right) p_{1}+C_{f} p_{3} .
\end{aligned}
$$


$p_{j}(j=1, \ldots, 4)$ denotes the probability of the occurrence of the event $\mathrm{E}_{\mathrm{j}}$. The length of a replacement cycle can be formulated to be

$$
V=T\left[I\left(\mathrm{E}_{1}\right)+I\left(\mathrm{E}_{3}\right)\right]+b I\left(\mathrm{E}_{2}\right)+R I\left(\mathrm{E}_{4}\right) .
$$

Hence, the expected length of a replacement cycle is

$$
E[V]=E[T I(0<T \leq b)]+b p_{2}+E\left[T I\left(X_{b}<\vartheta, b<T \leq R\right)\right]+E\left[R I\left(X_{b}<\vartheta, T>R\right)\right]
$$

In each replacement cycle, one of the four exclusive events occurs. In the long run, the evolution of the replacement cycle forms a stochastic process which will settle into equilibrium (Karlin and Taylor, 1975); the four basic events will occur with relative frequencies given by their respective probabilities. Therefore, the long-run cost per unit time, denoted by $c(b, \vartheta)$, is

$$
c(b, \vartheta)=\frac{E[C]}{E[V]} .
$$

The remaining task is to minimize the long-run cost per unit time by appropriately choosing the condition-monitoring epoch $b$ and the critical threshold $\vartheta$. It is evident that setting $b$ too small will result in a poor estimation of the preventive-replacement time, and that setting $b$ too large will risk unexpected failures before the inspection. Similarly, setting $\vartheta$ too small will result in too many premature replacements, and setting $\vartheta$ too large will risk unexpected failures after the inspection. Therefore, there is an economic necessity to minimize the long-run cost per unit time $c(b, \vartheta)$. In Section 3, we will elaborate the inspection-replacement policy by considering two widely used stochastic processes: the gamma process and the Wiener process.

Remark 1. It is worth noting that the inspection-replacement policy is targeted at individual components in the sense that the preventive-replacement time is not identical but altering among components. Only the degradation measurement at time $b$ is obtained, can the preventive-replacement time be determined. One may say that a more cost-efficient technique is to include the preventive-replacement time in the decision variables, and minimize the cost rate function by appropriately choosing $b, \vartheta$ and $R$ simultaneously. However, if we include $R$ in the decision variables, the preventive-replacement time will be identical for all the components in the heterogeneous population. Otherwise, if we want to find the optimal preventivereplacement time for each individual component, then the condition-monitoring epoch and the critical threshold must be pre-fixed. We cannot find the optimal preventive-replacement time for an individual component without knowing any degradation information of the component. Therefore, to schedule the optimal preventive-replacement time for each individual component, we cannot include $R$ in the decision variables.

To demonstrate the advantage of the inspection-replacement policy, we compare it with a joint policy which was advocated by Ye et al. (2012). The joint policy is composed of two successive procedures: a burn-in test by which weak components will be screened out, and then an age-based replacement by which burnt-in in-use components will be preventively replaced. Specifically, components in a heterogeneous population are all subject to a burn-in test with duration $\ddot{b}(>0)$. After the burn-in test, the deterioration level of each component is measured. Components with deterioration level exceeding the cut-off level $\ddot{\vartheta}(<l)$ are treated like weak and discarded. The burnt-in components, with deterioration level smaller than the cut-off level, 


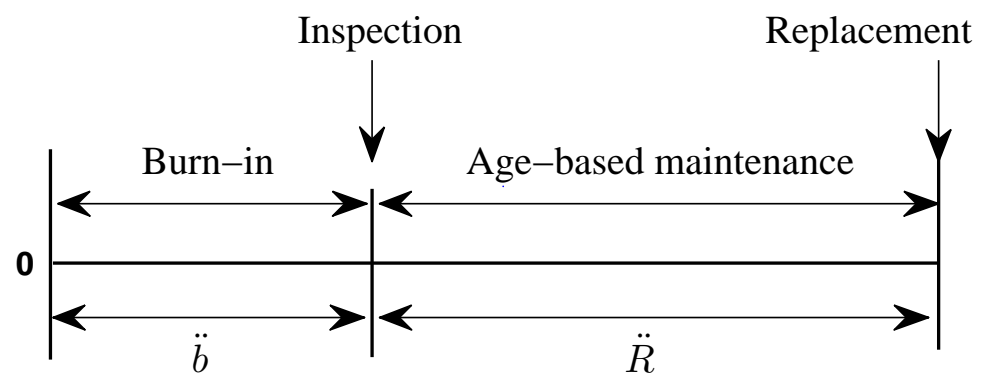

Figure 2: Schematic of the joint policy.

are put into field use and are subject to an age-based replacement: A burnt-in in-use component will be replaced by a new burnt-in component upon failure or at a pre-determined preventivereplacement time $\ddot{R}(>0)$, whichever occurs first (see Figure 2). The decision variables of the joint policy are the burn-in duration, $\ddot{b}$, the cut-off level, $\ddot{\vartheta}$, and the preventive-replacement time, $\ddot{R}$. The per-component burn-in costs include the unit-time burn-in cost, $C_{1}$, the manufacturing cost, $C_{2}$, the fixed burn-in cost, $C_{3}$, and the inspection cost, $C_{I}$. Let $C_{\ddot{b}, \ddot{\vartheta}}$ denote the amount of money a manufacture needs to pay in order to obtain an accepted burnt-in component. In the field operation, the replacement cost is therefore $C_{r}-C_{2}+C_{\ddot{b}, \ddot{\vartheta}}$. The failure cost is $C_{f}$ for each burnt-in component. Here, $C_{I}, C_{r}$ and $C_{f}$ are identical to what have been defined in the inspection-replacement policy. The objective is to minimize the long-run cost per unit time by appropriately choosing $\ddot{b}, \ddot{\vartheta}$ and $\ddot{R}$.

The inspection-replacement policy and the joint policy share some common ground. The objectives of the inspection at epoch $b$ and the burn-in test with duration $\ddot{b}$ are both to prevent early in-use failures. However, there are two essential differences between them. Firstly, the inspection-replacement policy is implemented in the field operation while the burn-in test is a process by which components are exercised prior to being placed in service. Secondly, the preventive-replacement time $\ddot{R}$ of the joint policy is identical for all the burnt-in components. The preventive-replacement time $R$ of the inspection-replacement policy is dynamically determined, varying among the components. One deficiency of the joint policy is that it only takes into account the cost criterion yet not the reliability criterion. The inspection-replacement policy, however, takes into account the cost and reliability simultaneously. The preventivereplacement time $R$ is determined in a way such that the reliability of the functioning component is guaranteed to be larger than the threshold $\delta$.

\section{APPLIED TO TWO SPECIFIC PROCESSES}

In this section we draw the advanced inspection-replacement policy on two predominant stochastic processes: the gamma process and the Wiener process. The gamma process is the limit of a shot-noise process with exponential decay, and the wear is non-decreasing. The Wiener process is an almost surely continuous martingale. The gamma process and the Wiener process can be visualized in different contexts and have been studied rather extensively; see Balka et al. (2009) and van Noortwijk (2009) for review. 


\subsection{Strategy for Gamma Process}

The gamma process is a stochastic process with independent, gamma-distributed increments. The gamma process is suitable for modelling gradual damage monotonically accumulating over time in a sequence of tiny increments, such as wear, fatigue, corrosion, etc. Another advantage of the gamma process is the existence of an explicit probability density function (PDF), which permits feasible mathematical developments (van Noortwijk, 2009). Maintenance strategies developed in the literature when the deterioration follows the gamma process can be found in Castro (2013), Cheng et al. (2012) and Huynh et al. (2012). Let $v(t)$ be a non-decreasing, rightcontinuous, real-valued function on $[0,+\infty)$, with $v(0)=0$. Roughly speaking, a continuoustime stochastic process $\left\{Z_{t}, t \geq 0\right\}$ is a gamma process such that, for all $0 \leq s<t$, the random increment $Z_{t}-Z_{s}$ is independent of $Z_{s}$ and has a gamma distribution $G(v(t)-v(s), \beta)$. v(t)$v(s)$ is the shape parameter. $\beta(>0)$ is the scale parameter. The marginal density function of $\left\{Z_{t}, t \geq 0\right\}$ at time $t$ is

$$
g(z ; v(t))=P\left(Z_{t}=z\right)=\frac{\beta^{v(t)}}{\Gamma(v(t))} z^{v(t)-1} \exp (-\beta z), \quad z \geq 0 .
$$

Here, $\Gamma(a)=\int_{0}^{\infty} z^{a-1} \exp (-z) d z$ is the gamma function for $a>0$. The first hitting time of the process $\left\{Z_{t}, t \geq 0\right\}$ to the failure threshold $l$ has the cumulative distribution function (CDF):

$$
\begin{aligned}
F_{G a}(t ; v(t), l) & =P\left(Z_{t} \geq l\right)=\int_{l}^{\infty} P\left(Z_{t}=z\right) d z \\
& =\int_{l}^{\infty} \frac{\beta^{v(t)}}{\Gamma(v(t))} z^{v(t)-1} \exp (-\beta z) d z=\int_{\beta l}^{\infty} \frac{z^{v(t)-1}}{\Gamma(v(t))} \exp (-z) d z \\
& =\frac{\Gamma(v(t), \beta l)}{\Gamma(v(t))} .
\end{aligned}
$$

Here, $\Gamma(a, \tau)=\int_{\tau}^{\infty} z^{a-1} \exp (-z) d z$ is the incomplete gamma function for $a>0$ and $\tau \geq 0$. On one hand, the PDF of the first hitting time, by using the chain rule for differentiation, is

$$
\begin{aligned}
f_{G a}(t ; v(t), l) & =\frac{\partial}{\partial t} F_{G a}(t ; v(t), l) \\
& =\frac{v^{\prime}(t)}{\Gamma(v(t))} \int_{\beta l}^{\infty}[\log (z)-\psi(v(t))] z^{v(t)-1} \exp (-z) d z .
\end{aligned}
$$

Here, $\psi(a)=\Gamma^{\prime}(a) / \Gamma(a)$ is the digamma function for $a>0$. On the other hand, because the gamma process is monotonically increasing, the PDF of the first hitting time can be re-written by

$$
f_{G a}(t ; v(t), l)=P\left(Z_{t}=l\right)=g(l ; v(t)) .
$$

Therefore, $\forall z>0$, we can conclude that

$$
g(z ; v(t))=\frac{\partial}{\partial t} F_{G a}(t ; v(t), z) .
$$

We assume that the deterioration of a component from the normal sub-population follows the gamma process with marginal distribution $G\left(\alpha_{1} t, \beta\right)$. The deterioration of a component from the weak sub-population follows the gamma process with marginal distribution 
$G\left(\alpha_{2} t, \beta\right)$. We have $\alpha_{2}>\alpha_{1}>0$ and $\beta>0$. Hence, the marginal density function of the degradation process $\left\{X_{t}, t \geq 0\right\}$ at time $t$ is

$$
P\left(X_{t}=x\right)=w_{1} g\left(x ; \alpha_{1} t\right)+w_{2} g\left(x ; \alpha_{2} t\right), \quad x \geq 0 .
$$

Here, $w_{1}$ and $w_{2}$ are the respective proportions of the normal and the weak components in the heterogeneous population, with $w_{1}+w_{2}=1$. The parameters $\alpha_{i}, w_{i}(i=1,2)$ and $\beta$ can be estimated from degradation data and failure data by using classical parameter-estimation methods. Given that a component survives beyond the condition-monitoring epoch $b$ with degradation $X_{b}=x(<l)$, the probability of the component being normal is

$$
w_{1, X_{b}}=P\left(\text { normal } \mid T>b, X_{b}=x\right)=\frac{w_{1} g\left(x ; \alpha_{1} b\right)}{w_{1} g\left(x ; \alpha_{1} b\right)+w_{2} g\left(x ; \alpha_{2} b\right)} .
$$

The probability of the component being weak is

$$
w_{2, X_{b}}=P\left(\text { weak } \mid T>b, X_{b}=x\right)=\frac{w_{2} g\left(x ; \alpha_{2} b\right)}{w_{1} g\left(x ; \alpha_{1} b\right)+w_{2} g\left(x ; \alpha_{2} b\right)} .
$$

Therefore, for a component having survived beyond the condition-monitoring epoch $b$ with degradation $X_{b}=x$, the remaining useful life of the component has distribution function

$$
\begin{aligned}
P\left(T \leq t \mid T>b, X_{b}=x\right) & =P\left(X_{t-b} \geq l-x\right) \\
& =w_{1, X_{b}} \frac{\Gamma\left(\alpha_{1}(t-b), \beta(l-x)\right)}{\Gamma\left(\alpha_{1}(t-b)\right)}+w_{2, X_{b}} \frac{\Gamma\left(\alpha_{2}(t-b), \beta(l-x)\right)}{\Gamma\left(\alpha_{2}(t-b)\right)} .
\end{aligned}
$$

The preventive-replacement time $R$ should be scheduled such that $P\left(T \geq R \mid T>b, X_{b}=x\right)=$ $\delta$. Note that the complementary $\operatorname{CDF} P\left(T \geq t \mid T>b, X_{b}=x\right)$ is a monotonically decreasing function of $t$. Therefore, the preventive-replacement time $R$ can be uniquely determined. $R$ has to be calculated by using numerical methods. The calculation of $R$ is rather straightforward with today's computational capacity.

The probability of the occurrence of the event $E_{1}$ is

$$
p_{1}=P(0<T \leq b)=w_{1} F_{G a}\left(b ; \alpha_{1} b, l\right)+w_{2} F_{G a}\left(b ; \alpha_{2} b, l\right) .
$$

The probability of the occurrence of the event $\mathrm{E}_{3}$ is

$$
\begin{aligned}
p_{3}= & P\left(X_{b}<\vartheta, b<T \leq R\right) \\
= & \int_{0}^{\vartheta} w_{1} P\left(X_{R} \geq l \mid X_{b}=x, \text { normal }\right) P\left(X_{b}=x \mid \text { normal }\right) d x \\
& \quad+\int_{0}^{\vartheta} w_{2} P\left(X_{R} \geq l \mid X_{b}=x, \text { weak }\right) P\left(X_{b}=x \mid \text { weak }\right) d x \\
= & w_{1} \int_{0}^{\vartheta} F_{G a}\left(R-b ; \alpha_{1}(R-b),(l-x)\right) g\left(x ; \alpha_{1} b\right) d x \\
& \quad+w_{2} \int_{0}^{\vartheta} F_{G a}\left(R-b ; \alpha_{2}(R-b),(l-x)\right) g\left(x ; \alpha_{2} b\right) d x .
\end{aligned}
$$


The expectation $E[T I(0<T \leq b)]$ can be formulated to be

$$
\begin{aligned}
E[T I(0<T \leq b)]= & \int_{0}^{b} t P(T=t) d t=\int_{0}^{b} t P\left(X_{t}=l\right) d t \\
= & w_{1} \int_{0}^{b} t g\left(l ; \alpha_{1} t\right) d t+w_{2} \int_{0}^{b} t g\left(l ; \alpha_{2} t\right) d t \\
= & w_{1} \int_{0}^{b} t d F_{G a}\left(t ; \alpha_{1} t, l\right)+w_{2} \int_{0}^{b} t d F_{G a}\left(t ; \alpha_{2} t, l\right) \\
= & w_{1}\left[b F_{G a}\left(b ; \alpha_{1} b, l\right)-\int_{0}^{b} F_{G a}\left(t ; \alpha_{1} t, l\right) d t\right] \\
& \quad+w_{2}\left[b F_{G a}\left(b ; \alpha_{2} b, l\right)-\int_{0}^{b} F_{G a}\left(t ; \alpha_{2} t, l\right) d t\right] .
\end{aligned}
$$

The probability of the occurrence of the event $E_{2}$ is

$$
\begin{aligned}
p_{2}= & P\left(\vartheta \leq X_{b}<l\right) \\
= & w_{1}\left[F_{G a}\left(b ; \alpha_{1} b, \vartheta\right)-F_{G a}\left(b ; \alpha_{1} b, l\right)\right] \\
& \quad+w_{2}\left[F_{G a}\left(b ; \alpha_{2} b, \vartheta\right)-F_{G a}\left(b ; \alpha_{2} b, l\right)\right] .
\end{aligned}
$$

It can be evidenced that

$$
\begin{aligned}
E[T I(0<T \leq b)]+b p_{2}=w_{1} & {\left[b F_{G a}\left(b ; \alpha_{1} b, \vartheta\right)-\int_{0}^{b} F_{G a}\left(t ; \alpha_{1} t, l\right) d t\right] } \\
& +w_{2}\left[b F_{G a}\left(b ; \alpha_{2} b, \vartheta\right)-\int_{0}^{b} F_{G a}\left(t ; \alpha_{2} t, l\right) d t\right] .
\end{aligned}
$$

The expectation $E\left[T I\left(X_{b}<\vartheta, b<T \leq R\right)\right]$ can be formulated to be

$$
\begin{aligned}
E\left[T I\left(X_{b}<\vartheta, b<T \leq R\right)\right]= & \int_{0}^{\vartheta} \int_{b}^{R} t P\left(T=t \mid X_{b}=x\right) P\left(X_{b}=x\right) d t d x \\
= & \int_{0}^{\vartheta} \int_{b}^{R} t P\left(X_{t-b}=l-x\right) P\left(X_{b}=x\right) d t d x \\
= & w_{1} \int_{0}^{\vartheta} \int_{b}^{R} \operatorname{tg}\left(l-x ; \alpha_{1}(t-b)\right) g\left(x ; \alpha_{1} b\right) d t d x \\
& \quad+w_{2} \int_{0}^{\vartheta} \int_{b}^{R} \operatorname{tg}\left(l-x ; \alpha_{2}(t-b)\right) g\left(x ; \alpha_{2} b\right) d t d x .
\end{aligned}
$$

Because, for $i=1,2$,

$$
\begin{aligned}
\int_{b}^{R} \operatorname{tg}\left(l-x ; \alpha_{i}(t-b)\right) g\left(x ; \alpha_{i} b\right) d t & =g\left(x ; \alpha_{i} b\right) \int_{b}^{R} \operatorname{tg}\left(l-x ; \alpha_{i}(t-b)\right) d t \\
& =g\left(x ; \alpha_{i} b\right) \int_{b}^{R} t d F_{G a}\left(t-b ; \alpha_{i}(t-b),(l-x)\right),
\end{aligned}
$$

and

$$
\begin{aligned}
\int_{b}^{R} t d F_{G a}\left(t-b ; \alpha_{i}(t-b),(l-x)\right)= & R F_{G a}\left(R-b ; \alpha_{i}(R-b),(l-x)\right) \\
& -\int_{b}^{R} F_{G a}\left(t-b ; \alpha_{i}(t-b),(l-x)\right) d t,
\end{aligned}
$$


we have

$$
\begin{aligned}
E\left[T I\left(X_{b}<\vartheta, b<T \leq R\right)\right]= & w_{1} \int_{0}^{\vartheta} R F_{G a}\left(R-b ; \alpha_{1}(R-b),(l-x)\right) g\left(x ; \alpha_{1} b\right) d x \\
& -w_{1} \int_{0}^{\vartheta} \int_{b}^{R} F_{G a}\left(t-b ; \alpha_{1}(t-b),(l-x)\right) g\left(x ; \alpha_{1} b\right) d t d x \\
& +w_{2} \int_{0}^{\vartheta} R F_{G a}\left(R-b ; \alpha_{2}(R-b),(l-x)\right) g\left(x ; \alpha_{2} b\right) d x \\
& -w_{2} \int_{0}^{\vartheta} \int_{b}^{R} F_{G a}\left(t-b ; \alpha_{2}(t-b),(l-x)\right) g\left(x ; \alpha_{2} b\right) d t d x .
\end{aligned}
$$

The expectation $E\left[R I\left(X_{b}<\vartheta, T>R\right)\right]$ can be formulated to be

$$
\begin{aligned}
E\left[R I\left(X_{b}<\vartheta, T>R\right)\right]= & E\left[R I\left(X_{b}<\vartheta, X_{R}<l\right)\right] \\
= & \int_{0}^{\vartheta} R P\left(X_{R-b}<l-x\right) P\left(X_{b}=x\right) d x \\
= & w_{1} \int_{0}^{\vartheta} R\left[1-F_{G a}\left(R-b ; \alpha_{1}(R-b),(l-x)\right)\right] g\left(x ; \alpha_{1} b\right) d x \\
& \quad+w_{2} \int_{0}^{\vartheta} R\left[1-F_{G a}\left(R-b ; \alpha_{2}(R-b),(l-x)\right)\right] g\left(x ; \alpha_{2} b\right) d x .
\end{aligned}
$$

It can be evidenced that

$$
\begin{aligned}
& E\left[T I\left(X_{b}<\vartheta, b<T \leq R\right)\right]+E\left[R I\left(X_{b}<\vartheta, T>R\right)\right] \\
&= w_{1}\left[\int_{0}^{\vartheta} \operatorname{Rg}\left(x ; \alpha_{1} b\right) d x-\int_{0}^{\vartheta} \int_{b}^{R} F_{G a}\left(t-b ; \alpha_{1}(t-b),(l-x)\right) g\left(x ; \alpha_{1} b\right) d t d x\right] \\
&+w_{2}\left[\int_{0}^{\vartheta} \operatorname{Rg}\left(x ; \alpha_{2} b\right) d x-\int_{0}^{\vartheta} \int_{b}^{R} F_{G a}\left(t-b ; \alpha_{2}(t-b),(l-x)\right) g\left(x ; \alpha_{2} b\right) d t d x\right] .
\end{aligned}
$$

The integrations can be evaluated by using numerical methods. The cost rate function for the gamma process is readily obtained, substituting $p_{i}(i=1,2,3)$, Equation (1) and Equation (2) into $c(b, \vartheta)$. The minimal cost rate can be sought numerically.

\subsection{Strategy for Wiener Process}

The Wiener process $\left\{Z_{t}, t \geq 0\right\}$ with linear drift is defined by $Z_{t}=\eta t+\sigma B(t)$ in which $\eta(>0)$ is the drift parameter, and $\sigma(>0)$ is the variance coefficient (Singpurwalla, 1995). $B(t)$ is the standard Brownian motion. Maintenance strategies developed in the literature when the deterioration follows the Wiener process can be found in Barker and Newby (2009) and Guo et al. (2013). The Wiener process has independent and normally distributed increments; that is, for all $0 \leq s<t, Z_{t}-Z_{s}$ is independent of $Z_{s}$ and follows the normal distribution $N\left(\eta(t-s), \sigma^{2}(t-s)\right)$. Given the failure threshold $l$, the first hitting time conforms to the inverse Gaussian distribution with the PDF

$$
f_{I G}(t ; \mu, \lambda)=\left(\frac{\lambda}{2 \pi t^{3}}\right)^{1 / 2} \exp \left(-\frac{\lambda(t-\mu)^{2}}{2 \mu^{2} t}\right),
$$


and the CDF

$$
F_{I G}(t ; \mu, \lambda)=\Phi\left(\sqrt{\frac{\lambda}{t}}\left(\frac{t}{\mu}-1\right)\right)+\exp \left(\frac{2 \lambda}{\mu}\right) \Phi\left(-\sqrt{\frac{\lambda}{t}}\left(\frac{t}{\mu}+1\right)\right) .
$$

Here $\mu=l \times \eta^{-1}$, and $\sqrt{\lambda}=l \times \sigma^{-1} . \Phi(\cdot)$ is the CDF of the standard normal distribution.

The deterioration of a component from the normal sub-population is assumed to follow the Wiener process with drift parameter $\eta_{1}(>0)$ and variance coefficient $\sigma(>0)$. The deterioration of a component from the weak sub-population is assumed to follow the Wiener process with drift parameter $\eta_{2}\left(>\eta_{1}\right)$ and the same variance coefficient $\sigma$. Hence, the marginal density function of the degradation process $\left\{X_{t}, t \geq 0\right\}$ at time $t$ is

$$
\begin{aligned}
P\left(X_{t}=x\right) & =\frac{1}{\sqrt{2 \pi \sigma^{2} t}}\left[w_{1} \exp \left(-\frac{\left(x-\eta_{1} t\right)^{2}}{2 \sigma^{2} t}\right)+w_{2} \exp \left(-\frac{\left(x-\eta_{2} t\right)^{2}}{2 \sigma^{2} t}\right)\right] \\
& =\frac{w_{1}}{\sigma \sqrt{t}} \phi\left(\frac{x-\eta_{1} t}{\sigma \sqrt{t}}\right)+\frac{w_{2}}{\sigma \sqrt{t}} \phi\left(\frac{x-\eta_{2} t}{\sigma \sqrt{t}}\right),
\end{aligned}
$$

in which $\phi(\cdot)$ is the PDF of the standard normal distribution. $w_{1}$ and $w_{2}$ are the respective proportions of the normal and the weak components in the heterogeneous population, with $w_{1}+w_{2}=1$. The parameters $\eta_{i}, w_{i}(i=1,2)$ and $\sigma$ can be estimated from degradation data and failure data by using classical statistical methods. For notational convenience, define three functions of $x: \mu_{x}^{1}=(l-x) \times \eta_{1}^{-1}, \mu_{x}^{2}=(l-x) \times \eta_{2}^{-1}$ and $\lambda_{x}=(l-x)^{2} \times \sigma^{-2}$. The first hitting time of $\left\{X_{t}, t \geq 0\right\}$ to the failure threshold $l$ has a mixture distribution:

$$
P(T \leq t)=w_{1} F_{I G}\left(t ; \mu_{0}^{1}, \lambda_{0}\right)+w_{2} F_{I G}\left(t ; \mu_{0}^{2}, \lambda_{0}\right) .
$$

Note the Wiener process is not monotonically increasing. It is necessary to calculate $P(T>$ $\left.b, X_{b}=x\right)$ : the probability of a product surviving beyond time $b$ with the deterioration level at time $b$ being $x(<l)$. According to $\mathrm{Lu}(1995)$, the probability $P\left(T>b, X_{b}=x\right)$ can be calculated by using the following formula:

$$
\begin{aligned}
P\left(T>b, X_{b}=x\right) & =\left[1-\exp \left(-\frac{2 l(l-x)}{\sigma^{2} b}\right)\right]\left[\frac{w_{1}}{\sigma \sqrt{b}} \phi\left(\frac{x-\eta_{1} b}{\sigma \sqrt{b}}\right)+\frac{w_{2}}{\sigma \sqrt{b}} \phi\left(\frac{x-\eta_{2} b}{\sigma \sqrt{b}}\right)\right] \\
& =f(x, b)\left[\frac{w_{1}}{\sigma \sqrt{b}} \phi\left(\frac{x-\eta_{1} b}{\sigma \sqrt{b}}\right)+\frac{w_{2}}{\sigma \sqrt{b}} \phi\left(\frac{x-\eta_{2} b}{\sigma \sqrt{b}}\right)\right]
\end{aligned}
$$

in which

$$
f(x, b)=1-\exp \left(-\frac{2 l(l-x)}{\sigma^{2} b}\right) .
$$

Therefore, for all $t>b$, we have

$$
\begin{aligned}
P\left(T>t, X_{b}=x\right)= & P\left(T>b, X_{b}=x\right) P\left(T>t \mid T>b, X_{b}=x\right) \\
= & w_{1} P\left(T>b, X_{b}=x \mid \text { normal }\right) P\left(T_{b, x} \geq t-b \mid \text { normal }\right) \\
& \quad+w_{2} P\left(T>b, X_{b}=x \mid \text { weak }\right) P\left(T_{b, x} \geq t-b \mid \text { weak }\right) \\
= & w_{1} \frac{f(x, b)}{\sigma \sqrt{b}} \phi\left(\frac{x-\eta_{1} b}{\sigma \sqrt{b}}\right)\left[1-F_{I G}\left(t-b ; \mu_{x}^{1}, \lambda_{x}\right)\right] \\
& \quad+w_{2} \frac{f(x, b)}{\sigma \sqrt{b}} \phi\left(\frac{x-\eta_{2} b}{\sigma \sqrt{b}}\right)\left[1-F_{I G}\left(t-b ; \mu_{x}^{2}, \lambda_{x}\right)\right] .
\end{aligned}
$$


Here, $T_{b, x}$ denotes the first hitting time of the degradation process $\left\{X_{t}-x, t \geq b\right\}$ to the threshold $(l-x)$. We have

$$
P\left(T_{b, x} \geq t-b \mid \text { normal }\right)=1-F_{I G}\left(t-b ; \mu_{x}^{1}, \lambda_{x}\right),
$$

and

$$
P\left(T_{b, x} \geq t-b \mid \text { weak }\right)=1-F_{I G}\left(t-b ; \mu_{x}^{2}, \lambda_{x}\right) .
$$

Given a component having survived beyond the condition-monitoring epoch $b$ with deterioration $X_{b}=x(<\vartheta)$, the probability of the component being normal is

$$
w_{1, X_{b}}=P\left(\operatorname{normal} \mid T>b, X_{b}=x\right)=\frac{w_{1} \phi\left(\frac{x-\eta_{1} b}{\sigma \sqrt{b}}\right)}{w_{1} \phi\left(\frac{x-\eta_{1} b}{\sigma \sqrt{b}}\right)+w_{2} \phi\left(\frac{x-\eta_{2} b}{\sigma \sqrt{b}}\right)} .
$$

The probability of the component being weak is

$$
w_{2, X_{b}}=P\left(\operatorname{weak} \mid T>b, X_{b}=x\right)=\frac{w_{2} \phi\left(\frac{x-\eta_{2} b}{\sigma \sqrt{b}}\right)}{w_{1} \phi\left(\frac{x-\eta_{1} b}{\sigma \sqrt{b}}\right)+w_{2} \phi\left(\frac{x-\eta_{2} b}{\sigma \sqrt{b}}\right)} .
$$

Therefore, for a component having survived beyond the condition-monitoring epoch $b$ with degradation $X_{b}=x$, the remaining useful life of the component has distribution function

$$
P\left(T \leq t \mid T>b, X_{b}=x\right)=w_{1, X_{b}} F_{I G}\left(t-b ; \mu_{x}^{1}, \lambda_{x}\right)+w_{2, X_{b}} F_{I G}\left(t-b ; \mu_{x}^{2}, \lambda_{x}\right) .
$$

The preventive-replacement time $R$ can therefore be uniquely determined by solving the equation $P\left(T \geq R \mid T>b, X_{b}=x\right)=\delta$.

The probability of the occurrence of the event $E_{1}$ is

$$
p_{1}=P(0<T \leq b)=w_{1} F_{I G}\left(b ; \mu_{0}^{1}, \lambda_{0}\right)+w_{2} F_{I G}\left(b ; \mu_{0}^{2}, \lambda_{0}\right) .
$$

The probability of the occurrence of the event $E_{3}$ is

$$
\begin{aligned}
p_{3}= & \int_{-\infty}^{\vartheta}\left[P\left(T>b, X_{b}=x\right)-P\left(T>R, X_{b}=x\right)\right] d x \\
= & \int_{-\infty}^{\vartheta} P\left(T_{b, x}<R-b\right) P\left(T>b, X_{b}=x\right) d x \\
= & \frac{w_{1}}{\sigma \sqrt{b}} \int_{-\infty}^{\vartheta} f(x, b) F_{I G}\left(R-b ; \mu_{x}^{1}, \lambda_{x}\right) \phi\left(\frac{x-\eta_{1} b}{\sigma \sqrt{b}}\right) d x \\
& \quad+\frac{w_{2}}{\sigma \sqrt{b}} \int_{-\infty}^{\vartheta} f(x, b) F_{I G}\left(R-b ; \mu_{x}^{2}, \lambda_{x}\right) \phi\left(\frac{x-\eta_{2} b}{\sigma \sqrt{b}}\right) d x \\
= & w_{1} \int_{0}^{\Phi\left(\frac{\vartheta-\eta_{1} b}{\sigma \sqrt{b}}\right)} f\left(\varphi_{1}(z), b\right) F_{I G}\left(R-b ; \mu_{\varphi_{1}(z)}^{1}, \lambda_{\varphi_{1}(z)}\right) d z \\
& \quad+w_{2} \int_{0}^{\Phi\left(\frac{\vartheta-\eta_{2} b}{\sigma \sqrt{b}}\right)} f\left(\varphi_{2}(z), b\right) F_{I G}\left(R-b ; \mu_{\varphi_{2}(z)}^{2}, \lambda_{\varphi_{2}(z)}\right) d z .
\end{aligned}
$$


The function $\varphi_{i}(\cdot)(i=1,2)$ is defined by

$$
\varphi_{i}(z)=\sigma \sqrt{b} \Phi^{-1}(z)+\eta_{i} b .
$$

Note that, when calculating $p_{3}$, we convert the problem of integration over an infinite interval to the problem of integration over a finite interval. If the integral is evaluated numerically, the conversion can reduce the computational load and improve the integral accuracy dramatically. The expectation $E[T I(0<T \leq b)]$ can be formulated to be

$$
\begin{aligned}
E[T I(0<T \leq b)]= & \int_{0}^{b} t\left[w_{1} f_{I G}\left(t ; \mu_{0}^{1}, \lambda_{0}\right)+w_{2} f_{I G}\left(t ; \mu_{0}^{2}, \lambda_{0}\right)\right] d t \\
= & \frac{l w_{1}}{\eta_{1}}\left[\Phi\left(-\frac{l-\eta_{1} b}{\sigma \sqrt{b}}\right)-\exp \left(\frac{2 \eta_{1} l}{\sigma^{2}}\right) \Phi\left(-\frac{l+\eta_{1} b}{\sigma \sqrt{b}}\right)\right] \\
& \quad+\frac{l w_{2}}{\eta_{2}}\left[\Phi\left(-\frac{l-\eta_{2} b}{\sigma \sqrt{b}}\right)-\exp \left(\frac{2 \eta_{2} l}{\sigma^{2}}\right) \Phi\left(-\frac{l+\eta_{2} b}{\sigma \sqrt{b}}\right)\right] .
\end{aligned}
$$

The derivation of $E[T I(0<T \leq b)]$ is given in Appendix A. The probability of the occurrence of the event $\mathrm{E}_{2}$ is

$$
p_{2}=P\left(T>b, X_{b} \geq \vartheta\right)=\int_{\vartheta}^{l} P\left(T>b, X_{b}=x\right) d x .
$$

The expectation $E\left[T I\left(X_{b}<\vartheta, b<T \leq R\right)\right]$ can be formulated to be

$$
\begin{aligned}
E\left[T I\left(X_{b}<\vartheta, b<T \leq R\right)\right] \\
=\int_{-\infty}^{\vartheta}\left[-\int_{b}^{R} t d P\left(T>t, X_{b}=x\right)\right] d x \\
=\int_{-\infty}^{\vartheta} b P\left(T>b, X_{b}=x\right) d x-\int_{-\infty}^{\vartheta} R P\left(T>R, X_{b}=x\right) d x \\
\quad+\frac{w_{2}}{\sigma \sqrt{b}} \int_{-\infty}^{\vartheta} \int_{b}^{R} f(x, b)\left[1-F_{I G}\left(t-b ; \mu_{x}^{2}, \lambda_{x}\right)\right] \phi\left(\frac{x-\eta_{2} b}{\sigma \sqrt{b}}\right) d t d x \\
\quad+\frac{w_{1}}{\sigma \sqrt{b}} \int_{-\infty}^{\vartheta} \int_{b}^{R} f(x, b)\left[1-F_{I G}\left(t-b ; \mu_{x}^{1}, \lambda_{x}\right)\right] \phi\left(\frac{x-\eta_{1} b}{\sigma \sqrt{b}}\right) d t d x .
\end{aligned}
$$

The expectation $E\left[R I\left(X_{b}<\vartheta, T>R\right)\right]$ can be formulated to be

$$
E\left[R I\left(X_{b}<\vartheta, T>R\right)\right]=\int_{-\infty}^{\vartheta} R P\left(T>R, X_{b}=x\right) d x .
$$

It can be evidenced that

$$
\begin{aligned}
b p_{2}+ & E\left[T I\left(X_{b}<\vartheta, b<T \leq R\right)\right]+E\left[R I\left(X_{b}<\vartheta, T>R\right)\right] \\
= & b \int_{-\infty}^{l} P\left(T>b, X_{b}=x\right) d x \\
& +\frac{w_{2}}{\sigma \sqrt{b}} \int_{-\infty}^{\vartheta} \int_{b}^{R} f(x, b)\left[1-F_{I G}\left(t-b ; \mu_{x}^{2}, \lambda_{x}\right)\right] \phi\left(\frac{x-\eta_{2} b}{\sigma \sqrt{b}}\right) d t d x \\
& +\frac{w_{1}}{\sigma \sqrt{b}} \int_{-\infty}^{\vartheta} \int_{b}^{R} f(x, b)\left[1-F_{I G}\left(t-b ; \mu_{x}^{1}, \lambda_{x}\right)\right] \phi\left(\frac{x-\eta_{1} b}{\sigma \sqrt{b}}\right) d t d x
\end{aligned}
$$


which can be further simplified into

$$
\begin{aligned}
b p_{2}+ & E\left[T I\left(X_{b}<\vartheta, b<T \leq R\right)\right]+E\left[R I\left(X_{b}<\vartheta, T>R\right)\right] \\
= & b w_{1}\left[\Phi\left(\frac{l-\eta_{1} b}{\sigma \sqrt{b}}\right)-\exp \left(\frac{2 \eta_{1} l}{\sigma^{2}}\right) \Phi\left(-\frac{l+\eta_{1} b}{\sigma \sqrt{b}}\right)\right] \\
& +b w_{2}\left[\Phi\left(\frac{l-\eta_{2} b}{\sigma \sqrt{b}}\right)-\exp \left(\frac{2 \eta_{2} l}{\sigma^{2}}\right) \Phi\left(-\frac{l+\eta_{2} b}{\sigma \sqrt{b}}\right)\right] \\
& +w_{1} \int_{0}^{\Phi\left(\frac{\vartheta-\eta_{1} b}{\sigma \sqrt{b}}\right)} \int_{b}^{R} f\left(\varphi_{1}(z), b\right)\left[1-F_{I G}\left(t-b ; \mu_{\varphi_{1}(z)}^{1}, \lambda_{\varphi_{1}(z)}\right)\right] d t d z \\
& +w_{2} \int_{0}^{\Phi\left(\frac{\vartheta-\eta_{2} b}{\sigma \sqrt{b}}\right)} \int_{b}^{R} f\left(\varphi_{2}(z), b\right)\left[1-F_{I G}\left(t-b ; \mu_{\varphi_{2}(z)}^{2}, \lambda_{\varphi_{2}(z)}\right)\right] d t d z .
\end{aligned}
$$

The cost rate function for the Wiener process is readily obtained, substituting $p_{i}(i=1,2,3)$, Equation (4) and Equation (5) into $c(b, \vartheta)$. The optimal condition-monitoring epoch and critical threshold can therefore be determined by minimizing the cost rate function.

\section{NUMERICAL EXAMPLES}

\subsection{Application: GaAs Lasers}

As a laser device ages, its light output decreases. Increasing a laser device's operating current may maintain its light output nearly constant. Therefore, the operating current usually serves as a performance characteristic for laser devices. A laser device is considered to have failed if its operating current is too high and crosses a threshold. Figure 3 shows the percent increase in operating current over time for a sample of fifteen GaAs laser devices (Meeker and Escobar. 1998). The GaAs laser devices were tested at $80^{\circ} \mathrm{C}$. After the installation of a new GaAs laser device, its operating current was measured every 250 hours. The experiment was terminated after 4000 hours. A GaAs laser device is considered to have failed if the percent increase in its operating current reaches the failure threshold $l=10$. That is, if the increase in its operating current is larger than 10 percent, a device is considered to have failed. It can be observed in Figure 3 that the operating current increases linearly with time and that four GaAs laser devices are non-conforming, deteriorating faster than the others. Therefore, the mixture gamma process and the mixture Wiener process are suitable for modeling the evolving process of the operating current. Since the operating current was measured every 250 hours, for ease of exposition, in what follows the unit of time is always set by 250 hours. By fitting the mixture gamma process and the mixture Wiener process to the GaAs laser data, the maximum likelihood estimates are given in Table 1. The maximized log-likelihoods, denoted by $\ell$, are also given. According to

Table 1: Maximum likelihood estimate with corresponding log-likelihood.

\begin{tabular}{ccc}
\hline Model & Maximum likelihood estimate & $\ell$ \\
\hline gamma & $\left\{\hat{w}_{2}, \hat{\alpha}_{1}, \hat{\alpha}_{2}, \hat{\beta}\right\}=\{0.2646,8.6129,12.9727,19.1764\}$ & 97.1152 \\
Wiener & $\left\{\hat{w}_{2}, \hat{\eta}_{1}, \hat{\eta}_{2}, \hat{\sigma}\right\}=\{0.2155,0.4563,0.7022,0.1727\}$ & 73.6541 \\
\hline
\end{tabular}

the maximized log-likelihoods, the mixture gamma process fits the GaAs laser data better than 




Figure 3: Plot of percent increase in operating current for GaAs lasers tested at $80^{\circ} \mathrm{C}$.

the mixture Wiener process. Therefore, we might treat the mixture gamma process to be the underlying stochastic law.

We implement the inspection-replacement policy on the GaAs laser devices to illustrate the procedure for determining the optimal condition-monitoring epoch and the optimal critical threshold. The mixture gamma process is assumed to be the underlying stochastic law with the parameters assuming the values estimated: $w_{2}=0.2646, \alpha_{1}=8.6129, \alpha_{2}=12.9727$, and $\beta=19.1764$. The cost configuration of the inspection-replacement policy is given: $C_{I}=1$, $C_{r}=50$, and $C_{f}=500$. The reliability threshold, i.e. $\delta$, is assigned with value 0.9 , such that devices will function with 0.1 probability of failure. Some direct-search techniques that do not require numerical or analytic gradients can be used to minimize the cost rate function. Representatives are the pattern search, the genetic algorithm and the Nelder-Mead method. In the following, we use the MATLAB function 'fminsearch' to minimize the cost rate function. We try various starting points to guarantee that the minimized cost rate is globally optimal. Under the above parameter setting and cost configuration, the optimal condition-monitoring time is $b^{*}=12.4776$. The optimal critical threshold is $\vartheta^{*}=6.6727$. The corresponding minimized long-run cost is $c^{*}=4.2188$ per unit time. The optimal inspection-replacement policy is hence carried out as follows. After the installation of a GaAs laser device, the operating current of the device will be measured at time $b^{*}=12.4776$. If the percent increase in operating current is larger than the critical threshold $\vartheta^{*}=6.6727$, the device will be treated like defective and immediately replaced by a new one. If the percent increase is smaller than the critical threshold, the device will be treated like normal and left untouched. The accepted 'normal' device will be preventively replaced at a specific preventive-replacement time point or upon failure, whichever occurs first. The preventive-replacement time is the time at which the reliability of the device drops to 0.9 . 
For illustrative purpose, we implement the inspection-replacement policy on other $24 \mathrm{GaAs}$ laser devices which are randomly sampled (simulated) from the heterogeneous population 1 . In Table 2, the devices highlighted in red are from the weak sub-population. The percent increase

Table 2: Implement the inspection-replacement policy on other 24 devices.

\begin{tabular}{ccccccccc}
\hline device & 1 & 2 & 3 & 4 & 5 & 6 & 7 & 8 \\
$X_{b^{*}}$ & 4.5650 & 5.9240 & 5.0045 & 5.7226 & 6.1297 & 6.6211 & 5.6950 & 5.2053 \\
$R$ & 23.1307 & 20.3083 & 22.2150 & 20.7248 & 19.8822 & 18.7485 & 20.7819 & 21.7975 \\
\hline device & 9 & 10 & 11 & 12 & 13 & 14 & 15 & 16 \\
$X_{b^{*}}$ & 5.8063 & 8.6244 & 6.0386 & 8.5035 & 5.8126 & 6.0069 & 5.2671 & 5.4225 \\
$R$ & 20.5517 & NA & 20.0711 & NA & 20.5386 & 20.1368 & 21.6689 & 21.3466 \\
\hline device & 17 & 18 & 19 & 20 & 21 & 22 & 23 & 24 \\
$X_{b^{*}}$ & 8.8244 & 5.4806 & 5.4592 & 5.8277 & 5.7365 & 8.4850 & 5.2647 & 7.3163 \\
$R$ & $\mathrm{NA}$ & 21.2259 & 21.2704 & 20.5074 & 20.6961 & $\mathrm{NA}$ & 21.6739 & $\mathrm{NA}$ \\
\hline
\end{tabular}

in operating current is recorded upon the optimal condition-monitoring time (see $X_{b^{*}}$ in Table 2). If the percent increase is larger than the optimal critical threshold, the device is immediately replaced by a new one (e.g., devices $10,12,17,22,24)$. If the percent increase is smaller than the critical threshold, a specific preventive-replacement time is scheduled for the device (see $R$ in Table 2). It can be seen in Table 2 that all the weak devices have been identified, showing the capability of the inspection-replacement policy in dealing with heterogeneous populations.

As a comparison, we implement the joint policy on the GaAs laser devices. The derivation of the cost rate function of the joint policy, when the deterioration follows the gamma process, is given in Appendix $\mathrm{B}$. The unit-time burn-in cost is $C_{1}=20$. The per-component manufacturing cost is $C_{2}=30$. The per-component fixed burn-in cost is $C_{3}=20$. By using the 'fminsearch' function, the optimal burn-in duration is $\ddot{b}^{*}=2.9735 \times 10^{-2}$. The optimal cut-off level is $\ddot{\vartheta}^{*}=0.4038$. The optimal preventive-replacement time is $\ddot{R}^{*}=12.6024$. The corresponding minimized long-run cost is $\ddot{c}^{*}=5.8827$ per unit time, which is larger than $c^{*}$. The larger $\ddot{c}^{*}$ evinces the feasibility and competence of the inspection-replacement policy. The optimal joint policy is hence carried out as follows. Put all the GaAs laser devices in a burn-in test with burn-in duration $\ddot{b}^{*}$. After the burn-in test, devices with percent increase in operating current larger than the cut-off level $\ddot{\vartheta}^{*}$ are discarded. Devices with percent increase smaller than the cut-off level are put into field operation. The accepted burnt-in devices will be preventively replaced at the preventive-replacement time point $\ddot{R}^{*}$ or upon failure, whichever occurs first.

Note that the optimal burn-in duration $\ddot{b}^{*}$ is much smaller than the optimal conditionmonitoring time $b^{*}$. The phenomenon indicates a fundamental difference between the inspectionreplacement policy and the joint policy: The inspection-replacement policy is advanced with the intention to take full advantage of weak components. Although the optimal preventivereplacement time $\ddot{R}^{*}$ is close to the optimal condition-monitoring time $b^{*}$, it is much smaller than the preventive-replacement time $R$ (see Table 2). Therefore, we say that the inspectionreplacement policy is able to yield much more income than the joint policy, by making the most of both weak components and normal components.

\footnotetext{
${ }^{1}$ Generate a random number from the binomial distribution with the number of trials being 1 and the probability of success being 0.7354 . If the generated number is 1 , we simulate $X_{b^{*}}$ from the normal sub-population. Otherwise, if the generated number is 0 , we simulate $X_{b^{*}}$ from the weak sub-population. Repeat the procedure for 24 times.
} 


\subsection{Simulation Study}

Having illustrated the procedure for determining the optimal condition-monitoring epoch and the optimal critical threshold, this section performs sensitivity analysis and contradistinctive analysis. Sensitivity analysis is performed to gain some insights into the impact of the weak proportion, $w_{2}$, on the optimal condition-monitoring epoch and the optimal critical threshold. Contradistinctive analysis is performed to indicate the conditions under which the inspectionreplacement policy is more cost-efficient than the joint policy.

\subsubsection{Gamma Process}

The parameter setting and cost configuration are identical to what have been given in Section 4.1: $\alpha_{1}=8.6129, \alpha_{2}=12.9727, \beta=19.1764, l=10, \delta=0.9, C_{1}=20, C_{2}=30, C_{3}=20$, $C_{I}=1, C_{r}=50$, and $C_{f}=500$. The degradation paths of twenty components, randomly drawn from a heterogeneous population (with $w_{2}=0.05$ ), are depicted in Figure 4 . It is observed that,



Figure 4: Gamma degradation paths with 20 entities.

in the drawn sample, two components are non-conforming, deteriorating faster than the others. We gradually increase the weak proportion $w_{2}$ from 0.02 to 0.28 with step size 0.02 . For each value of $w_{2}$, we seek for the minimal long-run cost per unit time of each policy. The optimal results of the inspection-replacement policy are summarized in Table 3. The optimal results of the joint policy are summarized in Table 4. In Table 3, the optimal condition-monitoring epoch is denoted by $b^{*}$. The optimal critical threshold is denoted by $\vartheta^{*}$. The minimized long-run cost per unit time is denoted by $c^{*}$. In Table 4 , the optimal burn-in duration is denoted by $\ddot{b}^{*}$. The optimal cut-off level is denoted by $\ddot{\vartheta}^{*}$. The optimal preventive-replacement time is denoted by $\ddot{R}^{*}$. The minimized long-run cost per unit time is denoted by $\ddot{c}^{*}$. 
Table 3: Optimal inspection-replacement policy for each value of $w_{2}$.

\begin{tabular}{cccccccc}
\hline$w_{2}$ & 0.02 & 0.04 & 0.06 & 0.08 & 0.10 & 0.12 & 0.14 \\
$b^{*}$ & 14.0990 & 13.5867 & 13.2754 & 13.0896 & 12.9590 & 12.8591 & 12.7787 \\
$\vartheta^{*}$ & 5.5844 & 5.6713 & 5.7900 & 6.3023 & 6.4911 & 6.4962 & 6.5264 \\
$c^{*}$ & 3.4736 & 3.9350 & 4.0045 & 4.0499 & 4.0836 & 4.1102 & 4.1322 \\
\hline$w_{2}$ & 0.16 & 0.18 & 0.20 & 0.22 & 0.24 & 0.26 & 0.28 \\
$b^{*}$ & 12.7117 & 12.6544 & 12.6045 & 12.5605 & 12.5210 & 12.4853 & 12.4528 \\
$\vartheta^{*}$ & 6.5380 & 6.6036 & 6.6486 & 6.6541 & 6.6602 & 6.6671 & 6.6842 \\
$c^{*}$ & 4.1509 & 4.1672 & 4.1815 & 4.1943 & 4.2059 & 4.2165 & 4.2262 \\
\hline
\end{tabular}

Table 4: Optimal joint policy for each value of $w_{2}$.

\begin{tabular}{cccccccc}
\hline$w_{2}$ & 0.02 & 0.04 & 0.06 & 0.08 & 0.10 & 0.12 & 0.14 \\
$\ddot{b}^{*}\left(\times 10^{-2}\right)$ & 3.3790 & 3.1052 & 3.0898 & 3.0774 & 3.0664 & 3.0583 & 3.0434 \\
$\ddot{\vartheta}^{*}$ & 0.3913 & 0.3933 & 0.3944 & 0.3952 & 0.3964 & 0.3972 & 0.3980 \\
$\ddot{R}^{*}$ & 14.2382 & 13.8991 & 13.4985 & 13.2799 & 13.13045 & 13.0641 & 12.9050 \\
$\ddot{c}^{*}$ & 4.6303 & 5.4555 & 5.5648 & 5.6340 & 5.6845 & 5.71 & 7.75 \\
\hline$w_{2}$ & 0.16 & 0.18 & 0.20 & 0.22 & 0.24 & 0.26 & 0.28 \\
$\ddot{b}^{*}\left(\times 10^{-2}\right)$ & 3.0352 & 3.0220 & 3.0115 & 2.9920 & 2.9858 & 2.9779 & 2.9683 \\
$\ddot{\vartheta}^{*}$ & 0.3992 & 0.3994 & 0.4010 & 0.4017 & 0.4025 & 0.4032 & 0.4045 \\
$\ddot{R}^{*}$ & 12.8561 & 12.7935 & 12.7392 & 12.7029 & 12.6663 & 12.6106 & 12.5823 \\
$\ddot{c}^{*}$ & 5.7840 & 5.8078 & 5.8287 & 5.8401 & 5.8593 & 5.8794 & 5.8896 \\
\hline
\end{tabular}

It is observed in Table 3 that, with the weak proportion increasing, the optimal conditionmonitoring epoch decreases, the optimal critical threshold increases, and the minimized cost rate increases. There would be more unexpected failures if the weak proportion increases, resulting in a higher expected cost. By bringing forward the inspection, we are able to prevent before-the-inspection failures. Therefore, a larger weak proportion results in a smaller optimal condition-monitoring epoch and a larger optimal cost rate. It is interesting to observe that an increasing weak proportion results in an increasing optimal critical threshold. One reasonable explanation is that the optimal critical threshold depends on the expected degradation at the condition-monitoring epoch. Because a weak component degrades faster than a normal component, the expected degradation at the same time point shall increase if the weak proportion increases. Therefore, a larger weak proportion results in a larger optimal critical threshold.

It is observed in Table 4 that, with the weak proportion increasing, the optimal burn-in duration decreases, the optimal cut-off level increases, the optimal preventive-replacement time decreases, and the optimal cost rate increases. The results in Table 4 are similar to the results in Table 3. Decreasing optimal burn-in duration corresponds to decreasing optimal conditionmonitoring epoch; increasing optimal cut-off level corresponds to increasing optimal critical threshold. With the weak proportion increasing, the weak components in the burnt-in population are expected to increase. Hence, the optimal preventive-replacement time is shortened to prevent costly in-use failures. As might have been expected, for each value of $w_{2}$, the optimal condition-monitoring epoch $b^{*}$ is close to the optimal preventive-replacement time $\ddot{R}^{*}$, but is much larger than the optimal burn-in duration $\ddot{b}^{*}$. By comparing $c^{*}$ and $\ddot{c}^{*}$, it is obvious that the inspection-replacement policy outperforms the joint policy, showing that the inspection- 
replacement policy is more cost-efficient.

One may argue that the reason for larger $\ddot{c}^{*}$ is that the joint policy includes two additional costs: the unit-time burn-in cost and the fixed burn-in cost. To reassure the sceptics, we set the unit-time burn-in cost and the fixed burn-in cost to be zero; that is $C_{1}=0$ and $C_{3}=0$. Under the circumstances, the inspection-replacement policy and the joint policy consume the same amount of money. With all the other costs and parameter values remain the same, we reminimize the cost rate function of the joint policy. The results are summarized in Table 5. With

Table 5: Optimal joint policy (with no burn-in costs) for each value of $w_{2}$.

\begin{tabular}{cccccccc}
\hline$w_{2}$ & 0.02 & 0.04 & 0.06 & 0.08 & 0.10 & 0.12 & 0.14 \\
$\ddot{b}^{*}\left(\times 10^{-2}\right)$ & 4.0857 & 3.8123 & 3.7796 & 3.7639 & 3.7540 & 3.7433 & 3.7304 \\
$\ddot{\vartheta}^{*}$ & 0.4803 & 0.4809 & 0.4814 & 0.4815 & 0.4817 & 0.4830 & 0.4833 \\
$\ddot{R}^{*}$ & 14.0559 & 13.5494 & 13.2305 & 13.0520 & 12.9219 & 12.7288 & 12.6857 \\
$\ddot{c}^{*}$ & 3.4835 & 3.9477 & 4.0184 & 4.0633 & 4.0972 & 4.1559 & 4.1654 \\
\hline$w_{2}$ & 0.16 & 0.18 & 0.20 & 0.22 & 0.24 & 0.26 & 0.28 \\
$\ddot{b}^{*}\left(\times 10^{-2}\right)$ & 3.7209 & 3.7171 & 3.7041 & 3.6828 & 3.6771 & 3.6644 & 3.6515 \\
$\ddot{\vartheta}^{*}$ & 0.4852 & 0.4869 & 0.4876 & 0.4884 & 0.4978 & 0.5010 & 0.5153 \\
$\ddot{R}^{*}$ & 12.6668 & 12.6131 & 12.5527 & 12.4871 & 12.4168 & 12.3492 & 12.2972 \\
$\ddot{c}^{*}$ & 4.1767 & 4.1939 & 4.2090 & 4.2118 & 4.2201 & 4.2307 & 4.2404 \\
\hline
\end{tabular}

the weak proportion increasing, the evolving trend of the optimal burn-in duration in Table 5 is identical to that in Table 4. So are the optimal cut-off level, the optimal preventive-replacement time and the minimized cost rate. For each value of $w_{2}$, the minimized cost rate in Table 5 is still larger than the minimized cost rate in Table 3. Table 5 shows that even if the burn-in test does not introduce any cost, the inspection-replacement policy is still superior to the joint policy. The reason for its superiority is that the inspection in the inspection-replacement policy and the burn-in test in the joint policy contribute equally: weeding out weak components. However, the preventive-replacement time $R$ is dynamically determined, depending on the physical degradation at time $b$ obtained by the inspection. In other words, the inspection-replacement policy takes full advantage of the information obtained by the inspection. By contrast, the preventive-replacement time $\ddot{R}$ is identical for all the burnt-in components. Although the physical degradation of each component is measured after the burn-in, it is not used for making maintenance decision. Indeed, $\ddot{R}$ is determined by only using the information on the original heterogeneous population. Therefore, the inspection-replacement policy dominates the joint policy.

\subsubsection{Wiener Process}

To show the wide applicability of the inspection-replacement policy, we apply it to the Wiener process. The following heterogeneous population will be investigated: $\eta_{1}=0.4563, \eta_{2}=$ 0.7022 , and $\sigma=0.1727$. The parameters are indeed assigned with the values estimated from the GaAs laser data. To be consistent, all the costs are identical to what have been given in Section 4.1. $C_{I}=1, C_{r}=50, C_{f}=500, C_{1}=20, C_{2}=30$ and $C_{3}=20$. The failure threshold and reliability threshold are, respectively, $l=10$ and $\delta=0.9$. The degradation paths of twenty components, randomly drawn from a heterogeneous population (with $w_{2}=0.05$ ), are depicted in Figure 5. It is observed that one component is non-conforming, deteriorating faster than the 


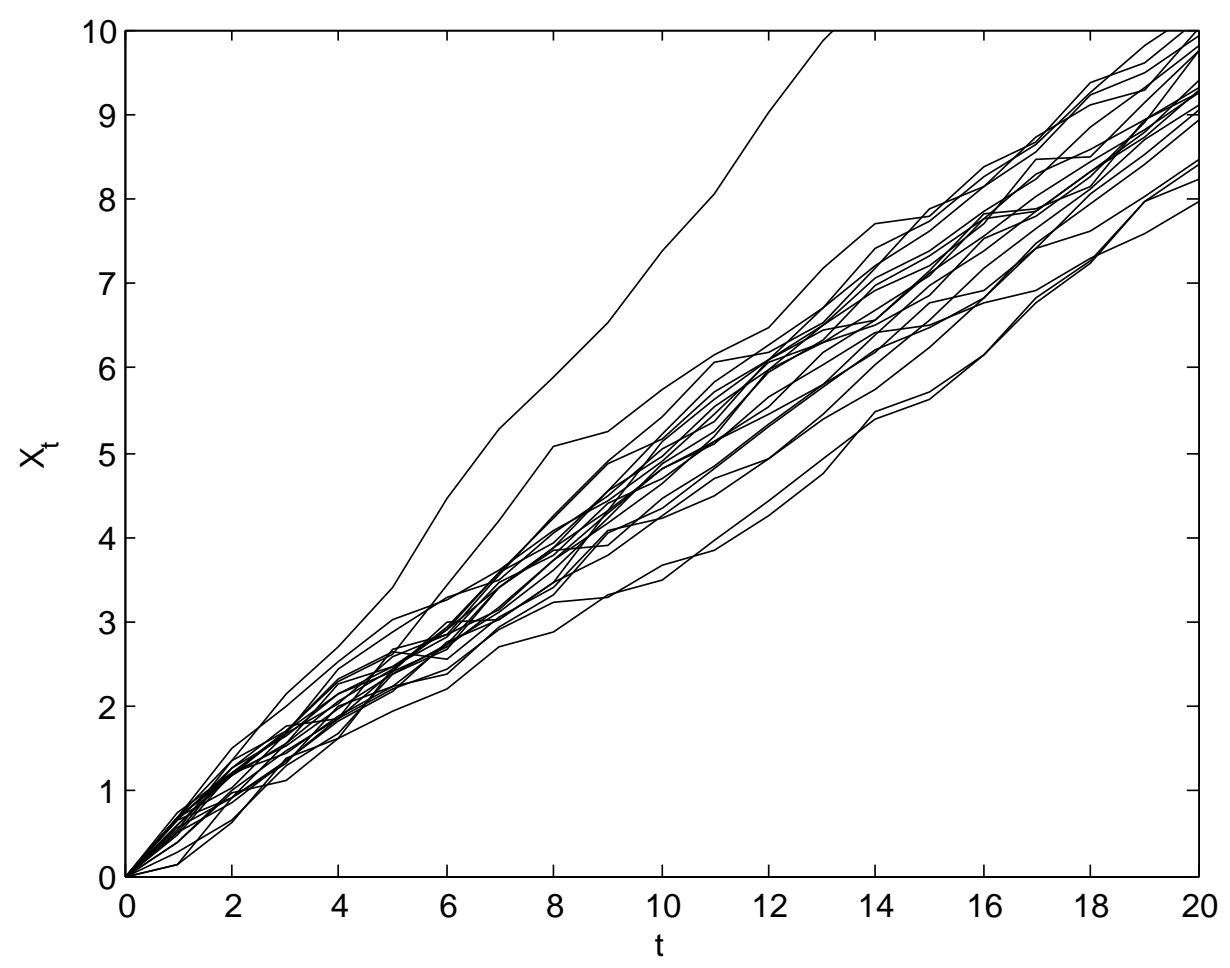

Figure 5: Wiener degradation paths with 20 entities.

others. We gradually increase the weak proportion $w_{2}$ from 0.02 to 0.28 with step size 0.02 . For each value of $w_{2}$, we seek for the minimal long-run cost per unit time of each policy. The derivation of the cost rate function of the joint policy, when the deterioration follows the Wiener process, is given in Appendix C. The optimal results of the inspection-replacement policy are summarized in Table 6. The optimal results of the joint policy are summarized in Table 7.

Table 6: Optimal inspection-replacement policy for each value of $w_{2}$.

\begin{tabular}{cccccccc}
\hline$w_{2}$ & 0.02 & 0.04 & 0.06 & 0.08 & 0.10 & 0.12 & 0.14 \\
$b^{*}$ & 13.7483 & 13.0596 & 12.8198 & 12.6757 & 12.5743 & 12.4965 & 12.4333 \\
$\vartheta^{*}$ & 5.5933 & 5.6293 & 5.7501 & 6.0196 & 6.1522 & 6.6139 & 6.8841 \\
$c^{*}$ & 3.9325 & 4.0543 & 4.1113 & 4.1483 & 4.1756 & 4.1970 & 4.2142 \\
\hline$w_{2}$ & 0.16 & 0.18 & 0.20 & 0.22 & 0.24 & 0.26 & 0.28 \\
$b^{*}$ & 12.3800 & 12.3341 & 12.2937 & 12.2577 & 12.2254 & 12.1960 & 12.1693 \\
$\vartheta^{*}$ & 7.1377 & 7.3107 & 7.3674 & 7.4329 & 7.4731 & 7.5427 & 7.6043 \\
$c^{*}$ & 4.2284 & 4.2403 & 4.2505 & 4.2593 & 4.2669 & 4.2737 & 4.2798 \\
\hline
\end{tabular}

It is observed that the results in Table 6 (Table 7) are in accordance with the results in Table 3 (Table 4). For the inspection-replacement policy, with the weak proportion increasing, the optimal condition-monitoring epoch decreases, the optimal critical threshold increases, and the minimized cost rate increases. For the joint policy, with the weak proportion increasing, the optimal burn-in duration decreases, the optimal cut-off level increases, the optimal preventivereplacement time decreases, and the optimal cost rate increases. The homology with the gamma process demonstrates the robustness of the inspection-replacement policy. As is obvious, when 
Table 7: Optimal joint policy for each value of $w_{2}$.

\begin{tabular}{cccccccc}
\hline$w_{2}$ & 0.02 & 0.04 & 0.06 & 0.08 & 0.10 & 0.12 & 0.14 \\
$\ddot{b}^{*}\left(\times 10^{-2}\right)$ & 5.3190 & 5.2284 & 5.1536 & 5.0352 & 4.8790 & 4.7794 & 4.6915 \\
$\ddot{\vartheta}^{*}$ & 0.5685 & 0.5748 & 0.5793 & 0.5834 & 0.5858 & 0.5893 & 0.5961 \\
$\ddot{R}^{*}$ & 13.2683 & 13.1030 & 13.0090 & 12.8419 & 12.7263 & 12.6395 & 12.5704 \\
$\ddot{c}^{*}$ & 4.7206 & 5.3048 & 5.6602 & 5.7161 & 5.7567 & 5.7885 & 5.8145 \\
\hline$w_{2}$ & 0.16 & 0.18 & 0.20 & 0.22 & 0.24 & 0.26 & 0.28 \\
$\ddot{b}^{*}\left(\times 10^{-2}\right)$ & 4.6104 & 4.5126 & 4.4427 & 4.3820 & 4.3093 & 4.2354 & 4.1497 \\
$\ddot{\vartheta}^{*}$ & 0.6006 & 0.6057 & 0.6121 & 0.6252 & 0.6333 & 0.6358 & 0.6400 \\
$\ddot{R}^{*}$ & 12.5132 & 12.4649 & 12.4229 & 12.3859 & 12.3529 & 12.3233 & 12.2962 \\
$\ddot{c}^{*}$ & 5.8365 & 5.8554 & 5.8721 & 5.8869 & 5.9003 & 5.9125 & 5.9236 \\
\hline
\end{tabular}

the deterioration follows the Wiener process, the inspection-replacement policy still outperforms the joint policy. To make the contradistinctive analysis sounder, we study the scenario in which the unit-time burn-in cost and the fixed burn-in cost are zero. The optimal results are given in Table 8. By comparing $\ddot{c}^{*}$ in Table 8 with $c^{*}$ in Table 6, the inspection-replacement

Table 8: Optimal joint policy (with no burn-in costs) for each value of $w_{2}$.

\begin{tabular}{cccccccc}
\hline$w_{2}$ & 0.02 & 0.04 & 0.06 & 0.08 & 0.10 & 0.12 & 0.14 \\
$\ddot{b}^{*}\left(\times 10^{-2}\right)$ & 5.3369 & 5.2603 & 5.1835 & 5.0503 & 4.9765 & 4.8850 & 4.8079 \\
$\ddot{\vartheta}^{*}$ & 0.5813 & 0.5875 & 0.5914 & 0.5968 & 0.6044 & 0.6082 & 0.6095 \\
$\ddot{R}^{*}$ & 13.2503 & 13.0582 & 12.8189 & 12.6746 & 12.5731 & 12.4959 & 12.4335 \\
$\ddot{c}^{*}$ & 3.9497 & 4.0676 & 4.1245 & 4.1616 & 4.1878 & 4.2103 & 4.2280 \\
\hline$w_{2}$ & 0.16 & 0.18 & 0.20 & 0.22 & 0.24 & 0.26 & 0.28 \\
$\ddot{b}^{*}\left(\times 10^{-2}\right)$ & 4.7143 & 4.6573 & 4.5834 & 4.4825 & 4.3990 & 4.3286 & 4.2435 \\
$\ddot{\vartheta}^{*}$ & 0.6155 & 0.6191 & 0.6243 & 0.6316 & 0.6370 & 0.6421 & 0.6497 \\
$\ddot{R}^{*}$ & 12.3813 & 12.3373 & 12.2988 & 12.2646 & 12.2341 & 12.2066 & 12.1816 \\
$\ddot{c}^{*}$ & 4.2429 & 4.2559 & 4.2673 & 4.2765 & 4.2867 & 4.2950 & 4.3027 \\
\hline
\end{tabular}

policy is undoubtedly superior to the joint policy. The contradistinctive analysis verifies the feasibility and efficiency of the inspection-replacement policy.

In real-life practice, engineers can make decisions based on the operating income as well as the burn-in costs. It should be noted that, with the weak proportion decreasing, the optimal critical threshold does not necessarily increase. Neither does the optimal burn-in duration. The relation between the optimal critical threshold (optimal burn-in duration) and the weak proportion is also controlled by other factors, e.g., the cost configuration. In real-life practice, the joint policy is suitable for immature products, whereas the inspection-replacement policy is suitable for both immature products and mature products. For immature products, the proportion of defective components is large. The burn-in procedure can be used to weed out most of the defective components. However, as a product's design, manufacturing, and testing processes mature, weak proportion in future generations of such product will decrease over time. When the proportion of defective components is small, the burn-in procedure is no longer costefficient. Because the inspection-replacement policy is also capable of weeding out defective components, it is suitable for both immature products and mature products. 


\section{CONCLUSIONS}

This study recommended an inspection-replacement policy for heterogeneous populations. The inspection-replacement policy possesses three main advantages. First, the inspection-replacement policy saves the burn-in costs. Second, the inspection-replacement policy makes full use of weak components. Third, the inspection-replacement policy takes into account the reliability criterion. Both sensitivity analysis and contradistinctive analysis were performed to demonstrate the feasibility and efficiency of the inspection-replacement policy. By comparing the minimized cost rates, it was shown that the inspection-replacement policy outperforms the joint policy. In real-life practice, the joint policy is suitable for the launch of a newly developed product, whereas the inspection-replacement policy is cost-efficient for both immature and mature products.

Future research can be done in many directions:

- The reliability threshold, $\delta$, can be included into the decision variables. The impact of the reliability threshold on the optimal maintenance can be investigated.

- In real-life practice, failures may not be easily identified. A component can be declared as 'failed' as soon as a defect or an important deterioration is presented even if the component is still functioning. The study of the case in which the failure is non-self-announcing is of great interest. Moreover, future research can be continued by considering random failure threshold, since in practice the failure threshold may not be deterministic.

- The gamma process is an increasing stochastic process while the Wiener process is nonmonotone. When assuming the Wiener process to be the underlying degradation process, one might use the last exit time from a critical threshold rather than the first hitting time to a failure threshold. The last exit time can be employed in future study to determine maintenance actions.

\section{APPENDIX}

\section{A Deriving the expectation $E[T I(0<T \leq b)]$}

By definition, we have

$$
\begin{aligned}
E[T I(0<T \leq b)] & =\int_{0}^{b} \frac{l}{\sigma \sqrt{2 \pi t}} \exp \left(-\frac{(\eta t-l)^{2}}{2 \sigma^{2} t}\right) d t \\
& =\int_{0}^{\sqrt{\eta b}} \frac{2 l}{\sigma \sqrt{2 \pi \eta}} \exp \left(-\frac{(z-l / z)^{2}}{2 \sigma^{2} / \eta}\right) d z
\end{aligned}
$$


in which $z$ is defined by $z=\sqrt{\eta t}$. We might denote $z-l / z$ by $v: v=z-l / z$. Because $z>0$, we have $z=v / 2+\sqrt{l+v^{2} / 4}$ and

$$
\begin{aligned}
& \frac{2 l}{\sigma \sqrt{2 \pi \eta}} \int_{0}^{\sqrt{\eta b}} \exp \left(-\frac{(z-l / z)^{2}}{2 \sigma^{2} / \eta}\right) d z \\
& =\frac{l}{\sigma \sqrt{2 \pi \eta}} \int_{-\infty}^{\sqrt{\eta b}-l / \sqrt{\eta b}}\left(1+\frac{v}{\sqrt{4 l+v^{2}}}\right) \exp \left(-\frac{v^{2}}{2 \sigma^{2} / \eta}\right) d v \\
& =\frac{l}{\sigma \sqrt{2 \pi \eta}}\left[\int_{-\infty}^{\sqrt{\eta b}-l / \sqrt{\eta b}} \exp \left(-\frac{v^{2}}{2 \sigma^{2} / \eta}\right) d v+\int_{-\infty}^{\sqrt{\eta b}-l / \sqrt{\eta b}} \frac{v}{\sqrt{4 l+v^{2}}} \exp \left(-\frac{v^{2}}{2 \sigma^{2} / \eta}\right) d v\right] .
\end{aligned}
$$

On one hand, we have

$$
\frac{l}{\sigma \sqrt{2 \pi \eta}} \int_{-\infty}^{\sqrt{\eta b}-l / \sqrt{\eta b}} \exp \left(-\frac{v^{2}}{2 \sigma^{2} / \eta}\right) d v=\frac{l}{\eta} \Phi\left(-\frac{l-b \eta}{\sigma \sqrt{b}}\right) .
$$

On the other hand, we have

$$
\begin{aligned}
\int_{-\infty}^{\sqrt{\eta b}-l / \sqrt{\eta b}} & \frac{v}{\sqrt{4 l+v^{2}}} \exp \left(-\frac{v^{2}}{2 \sigma^{2} / \eta}\right) d v \\
= & \exp \left(\frac{2 l \eta}{\sigma^{2}}\right) \int_{-\infty}^{\sqrt{\eta b}-l / \sqrt{\eta b}} \frac{v}{\sqrt{4 l+v^{2}}} \exp \left(-\frac{v^{2}+4 l}{2 \sigma^{2} / \eta}\right) d v \\
= & \exp \left(\frac{2 l \eta}{\sigma^{2}}\right) \int_{-\infty}^{0} \frac{v}{\sqrt{4 l+v^{2}}} \exp \left(-\frac{v^{2}+4 l}{2 \sigma^{2} / \eta}\right) d v \\
& +\exp \left(\frac{2 l \eta}{\sigma^{2}}\right) \int_{0}^{\sqrt{\eta b}-l / \sqrt{\eta b}} \frac{v}{\sqrt{4 l+v^{2}}} \exp \left(-\frac{v^{2}+4 l}{2 \sigma^{2} / \eta}\right) d v
\end{aligned}
$$

By setting $s=\sqrt{4 l+v^{2}}$, we have

$$
\int_{-\infty}^{0} \frac{v}{\sqrt{4 l+v^{2}}} \exp \left(-\frac{v^{2}+4 l}{2 \sigma^{2} / \eta}\right) d v=\int_{\infty}^{\sqrt{4 l}} \exp \left(-\frac{s^{2}}{2 \sigma^{2} / \eta}\right) d s
$$

and

$$
\int_{0}^{\sqrt{\eta b}-l / \sqrt{\eta b}} \frac{v}{\sqrt{4 l+v^{2}}} \exp \left(-\frac{v^{2}+4 l}{2 \sigma^{2} / \eta}\right) d v=\int_{\sqrt{4 l}}^{\sqrt{4 l+(\sqrt{\eta b}-l / \sqrt{\eta b})^{2}}} \exp \left(-\frac{s^{2}}{2 \sigma^{2} / \eta}\right) d s .
$$

Therefore, we have

$$
\begin{aligned}
\frac{l}{\sigma \sqrt{2 \pi \eta}} & \int_{-\infty}^{\sqrt{\eta b}-l / \sqrt{\eta b}} \frac{v}{\sqrt{4 l+v^{2}}} \exp \left(-\frac{v^{2}}{2 \sigma^{2} / \eta}\right) d v \\
& =\frac{l}{\sigma \sqrt{2 \pi \eta}} \exp \left(\frac{2 l \eta}{\sigma^{2}}\right) \int_{\infty}^{\sqrt{4 l+(\sqrt{\eta b}-l / \sqrt{\eta b})^{2}}} \exp \left(-\frac{s^{2}}{2 \sigma^{2} / \eta}\right) d s \\
& =-\frac{l}{\eta} \exp \left(\frac{2 l \eta}{\sigma^{2}}\right) \Phi\left(-\frac{l+b \eta}{\sigma \sqrt{b}}\right) .
\end{aligned}
$$


Hence, we obtain

$$
E[T I(0<T \leq b)]=\frac{l}{\eta} \Phi\left(-\frac{l-b \eta}{\sigma \sqrt{b}}\right)-\frac{l}{\eta} \exp \left(\frac{2 l \eta}{\sigma^{2}}\right) \Phi\left(-\frac{l+b \eta}{\sigma \sqrt{b}}\right)
$$

\section{B Joint policy for the gamma process}

In the gamma case, the expected cost to obtain an accepted (i.e., $X_{\ddot{b}}<\ddot{\vartheta}$ ) burnt-in component is

$$
C_{\ddot{b}, \ddot{\vartheta}}=\frac{C_{1} \ddot{b}+C_{2}+C_{3}+C_{I}}{w_{1} \int_{0}^{\ddot{\vartheta}} g\left(x ; \alpha_{1} \ddot{b}\right) d x+w_{2} \int_{0}^{\ddot{\vartheta}} g\left(x ; \alpha_{2} \ddot{b}\right) d x} .
$$

For an accepted burnt-in component, the probability that it belongs to the normal sub-population is

$$
w_{1}^{\prime}=\frac{w_{1} \int_{0}^{\ddot{\vartheta}} g\left(x ; \alpha_{1} \ddot{b}\right) d x}{w_{1} \int_{0}^{\ddot{\vartheta}} g\left(x ; \alpha_{1} \ddot{b}\right) d x+w_{2} \int_{0}^{\ddot{\vartheta}} g\left(x ; \alpha_{2} \ddot{b}\right) d x} .
$$

The probability that it belongs to the weak sub-population is

$$
w_{2}^{\prime}=\frac{w_{2} \int_{0}^{\ddot{\vartheta}} g\left(x ; \alpha_{2} \ddot{b}\right) d x}{w_{1} \int_{0}^{\ddot{\vartheta}} g\left(x ; \alpha_{1} \ddot{b}\right) d x+w_{2} \int_{0}^{\ddot{\vartheta}} g\left(x ; \alpha_{2} \ddot{b}\right) d x} .
$$

The expected total cost incurred in a replacement cycle is

$$
\begin{aligned}
E[C]=C_{r}-C_{2}+C_{\ddot{b}, \ddot{\vartheta}} & +C_{f} w_{1}^{\prime} \int_{0}^{\ddot{\vartheta}} F_{G a}\left(\ddot{R} ; \alpha_{1} \ddot{R}, l-x\right) g\left(x ; \alpha_{1} \ddot{b}\right) d x \\
& +C_{f} w_{2}^{\prime} \int_{0}^{\ddot{\vartheta}} F_{G a}\left(\ddot{R} ; \alpha_{2} \ddot{R}, l-x\right) g\left(x ; \alpha_{2} \ddot{b}\right) d x .
\end{aligned}
$$

The expected length of a replacement cycle is

$$
\begin{aligned}
E[V]=w_{1}^{\prime} & \int_{0}^{\ddot{R}} \int_{0}^{\ddot{\vartheta}}\left[1-F_{G a}\left(t ; \alpha_{1} t, l-x\right)\right] g\left(x ; \alpha_{1} \ddot{b}\right) d x d t \\
& +w_{2}^{\prime} \int_{0}^{\ddot{R}} \int_{0}^{\ddot{\vartheta}}\left[1-F_{G a}\left(t ; \alpha_{2} t, l-x\right)\right] g\left(x ; \alpha_{2} \ddot{b}\right) d x d t .
\end{aligned}
$$

The cost rate function for the joint policy is simply

$$
c(\ddot{b}, \ddot{\vartheta}, \ddot{R})=\frac{E[C]}{E[V]} .
$$

\section{Joint policy for the Wiener process}

In the Wiener case, the expected cost to obtain an accepted (i.e., $T>\ddot{b}$ and $X_{\ddot{b}}<\ddot{\vartheta}$ ) burnt-in component is

$$
C_{\ddot{b}, \ddot{\vartheta}}=\frac{C_{1} \ddot{b}+C_{2}+C_{3}+C_{I}}{P\left(T>\ddot{b}, X_{\ddot{b}}<\ddot{\vartheta}\right)},
$$


in which we have

$$
\begin{aligned}
P\left(T>\ddot{b}, X_{\ddot{b}}<\ddot{\vartheta}\right)= & \int_{-\infty}^{\ddot{\vartheta}} P\left(T>\ddot{b}, X_{\ddot{b}}=x\right) d x \\
= & \int_{-\infty}^{\ddot{\vartheta}} f(x, \ddot{b}) \frac{w_{1}}{\sigma \sqrt{\ddot{b}}} \phi\left(\frac{x-\eta_{1} \ddot{b}}{\sigma \sqrt{\ddot{b}}}\right) d x+\int_{-\infty}^{\ddot{\vartheta}} f(x, \ddot{b}) \frac{w_{2}}{\sigma \sqrt{\ddot{b}}} \phi\left(\frac{x-\eta_{2} \ddot{b}}{\sigma \sqrt{\ddot{b}}}\right) d x \\
= & w_{1} \int_{0}^{\Phi\left(\frac{\ddot{\vartheta}-\eta_{1} \ddot{b}}{\sigma \sqrt{b}}\right)} f\left(\eta_{1} \ddot{b}+\sigma \sqrt{\ddot{b}} \Phi^{-1}(z), \ddot{b}\right) d z \\
& +w_{2} \int_{0}^{\Phi\left(\frac{\ddot{\vartheta}-\eta_{2} \ddot{b}}{\sigma \sqrt{b}}\right)} f\left(\eta_{2} \ddot{b}+\sigma \sqrt{\ddot{b}} \Phi^{-1}(z), \ddot{b}\right) d z .
\end{aligned}
$$

For an accepted burnt-in component, the probability that it belongs to the normal sub-population is

$$
w_{1}^{\prime}=\frac{w_{1}}{P\left(T>\ddot{b}, X_{\ddot{b}}<\ddot{\vartheta}\right)} \int_{0}^{\Phi\left(\frac{\ddot{\vartheta}-\eta_{1} \ddot{b}}{\sigma \sqrt{b}}\right)} f\left(\eta_{1} \ddot{b}+\sigma \sqrt{\ddot{b}} \Phi^{-1}(z), \ddot{b}\right) d z .
$$

The probability that it belongs to the weak sub-population is

$$
w_{2}^{\prime}=\frac{w_{2}}{P\left(T>\ddot{b}, X_{\ddot{b}}<\ddot{\vartheta}\right)} \int_{0}^{\Phi\left(\frac{\ddot{\vartheta}-\eta_{2} \ddot{b}}{\sigma \sqrt{b}}\right)} f\left(\eta_{2} \ddot{b}+\sigma \sqrt{\ddot{b}} \Phi^{-1}(z), \ddot{b}\right) d z .
$$

For ease of exposition, define a function on $t>0$ :

$Q(t)$

$$
\begin{aligned}
& =w_{1}^{\prime} \frac{\int_{0}^{\Phi\left(\frac{\ddot{\vartheta}-\eta_{1} \ddot{b}}{\sigma \sqrt{b}}\right)} f\left(\eta_{1} \ddot{b}+\sigma \sqrt{\ddot{b}} \Phi^{-1}(z), \ddot{b}\right)\left[1-F_{I G}\left(t ; \mu_{\eta_{1} \ddot{b}+\sigma \sqrt{\vec{b}} \Phi^{-1}(z)}^{1}, \lambda_{\eta_{1} \ddot{b}+\sigma \sqrt{\vec{b}} \Phi^{-1}(z)}\right)\right] d z}{\int_{0}^{\Phi\left(\frac{\ddot{\vartheta}-\eta_{1} \ddot{b}}{\sigma \sqrt{b}}\right)} f\left(\eta_{1} \ddot{b}+\sigma \sqrt{\ddot{b}} \Phi^{-1}(z), \ddot{b}\right) d z} \\
& +w_{2}^{\prime} \frac{\int_{0}^{\Phi\left(\frac{\ddot{\vartheta}-\eta_{2} \ddot{b}}{\sigma \sqrt{b}}\right)} f\left(\eta_{2} \ddot{b}+\sigma \sqrt{\ddot{b}} \Phi^{-1}(z), \ddot{b}\right)\left[1-F_{I G}\left(t ; \mu_{\eta_{2} \ddot{b}+\sigma \sqrt{\ddot{b}} \Phi^{-1}(z)}^{2}, \lambda_{\eta_{2} \ddot{b}+\sigma \sqrt{\vec{b}} \Phi^{-1}(z)}\right)\right] d z}{\int_{0}^{\Phi\left(\frac{\ddot{\vartheta}-\eta_{2} \ddot{b}}{\sigma \sqrt{b}}\right)} f\left(\eta_{2} \ddot{b}+\sigma \sqrt{\ddot{b}} \Phi^{-1}(z), \ddot{b}\right) d z} .
\end{aligned}
$$

The expected total cost incurred in a replacement cycle is

$$
E[C]=C_{r}-C_{2}+C_{\ddot{b}, \ddot{\vartheta}}+C_{f}\left[1-P\left(T>\ddot{b}+\ddot{R} \mid T>\ddot{b}, X_{\ddot{b}}<\ddot{\vartheta}\right)\right] .
$$

It can be evidenced that

$$
P\left(T>\ddot{b}+\ddot{R} \mid T>\ddot{b}, X_{\ddot{b}}<\ddot{\vartheta}\right)=Q(\ddot{R}) .
$$

The expected length of a replacement cycle is

$$
E[V]=\int_{0}^{\ddot{R}} P\left(T>\ddot{b}+t \mid T>\ddot{b}, X_{\ddot{b}}<\ddot{\vartheta}\right) d t=\int_{0}^{\ddot{R}} Q(t) d t .
$$




\section{REFERENCES}

R. Ahmad and S. Kamaruddin. An overview of time-based and condition-based maintenance in industrial application. Computers \& Industrial Engineering, 63(1):135-149, 2012.

J. Balka, A. F. Desmond, and P. D. McNicholas. Review and implementation of cure models based on first hitting times for Wiener processes. Lifetime Data Analysis, 15(2):147-176, 2009.

C. Barker and M. Newby. Optimal non-periodic inspection for a multivariate degradation model. Reliability Engineering \& System Safety, 94(1):33-43, 2009.

M. Berrade, P. Scarf, C. Cavalcante, and R. Dwight. Imperfect inspection and replacement of a system with a defective state: A cost and reliability analysis. Reliability Engineering \& System Safety, 120(0):80-87, 2013.

I. T. Castro. An age-based maintenance strategy for a degradation-threshold-shock-model for a system subjected to multiple defects. Asia-Pacific Journal of Operational Research, 30(05): 1350016, 2013.

J. H. Cha and M. Finkelstein. Burn-in and the performance quality measures in heterogeneous populations. European Journal of Operational Research, 210(2):273-280, 2011.

J. H. Cha and M. Finkelstein. The failure rate dynamics in heterogeneous populations. Reliability Engineering \& System Safety, 112(0):120-128, 2013.

J. H. Cha and M. S. Finkelstein. Stochastically ordered subpopulations and optimal burn-in procedure. IEEE Transactions on Reliability, 59(4):635-643, 2010.

T. Cheng, M. D. Pandey, and J. van der Weide. The probability distribution of maintenance cost of a system affected by the gamma process of degradation: Finite time solution. Reliability Engineering \& System Safety, 108(0):65-76, 2012.

1. Eriolu, M. Eriolu, and N. ali. Heterogeneous data modeling with two-component weibullpoisson distribution. Journal of Applied Statistics, 40(11):2451-2461, 2013.

C. Guo, W. Wang, B. Guo, and X. Si. A maintenance optimization model for mission-oriented systems based on wiener degradation. Reliability Engineering \& System Safety, 111(0):183194, 2013.

K. Huynh, I. Castro, A. Barros, and C. Brenguer. Modeling age-based maintenance strategies with minimal repairs for systems subject to competing failure modes due to degradation and shocks. European Journal of Operational Research, 218(1):140-151, 2012.

S. Karlin and H. M. Taylor. A first course in stochastic processes, Second Edition. New York: Academic Press, 1975.

S. M. A. Kazmi, M. Aslam, S. Ali, and N. Abbas. Selection of suitable prior for the bayesian mixture of a class of lifetime distributions under type-i censored datasets. Journal of Applied Statistics, 40(8):1639-1658, 2013. 
J. Lu. A reliability model based on degradation and lifetime data. Ph.D. thesis, McGill University, Montreal, Canada, 1995.

W. Q. Meeker and L. A. Escobar. Statistical Methods for Reliability Data. New York: John Wiley \& Sons, 1998.

W. Pearn, J. Hong, and Y. Tai. The burn-in test scheduling problem with batch dependent processing time and sequence dependent setup time. International Journal of Production Research, 51(6):1694-1706, 2013.

J. W. Post and A. Bhattacharyya. Burn-in and thermal cyclic tests to determine the short-term reliability of a thin film resistance temperature detector. Microelectronics Reliability, 52(7): 1389-1395, 2012.

P. A. Scarf and C. A. V. Cavalcante. Modelling quality in replacement and inspection maintenance. International Journal of Production Economics, 135(1):372-381, 2012.

N. Singpurwalla. Survival in dynamic environments. Statistical Science, 10(1):86-103, 1995.

C. C. Tsai, S. T. Tseng, and N. Balakrishnan. Optimal burn-in policy for highly reliable products using gamma degradation process. IEEE Transactions on Reliability, 60(1):234-245, 2011.

J. M. van Noortwijk. A survey of the application of gamma processes in maintenance. Reliability Engineering \& System Safety, 94(1):2-21, 2009.

Y. Xiang, D. W. Coit, and Q. Feng. n subpopulations experiencing stochastic degradation: reliability modeling, burn-in, and preventive replacement optimization. IIE Transactions, 45 (4):391-408, 2013.

Z. S. Ye, Y. Shen, and M. Xie. Degradation-based burn-in with preventive maintenance. European Journal of Operational Research, 221(2):360-367, 2012.

Z. S. Ye, L. C. Tang, and M. Xie. A burn-in scheme based on percentiles of the residual life. Journal of Quality Technology, 43(4):334-345, 2011.

Z. S. Ye, M. Xie, L. C. Tang, and Y. Shen. Degradation-based burn-in planning under competing risks. Technometrics, 54(2):159-168, 2012.

T. Yuan and Y. Kuo. Bayesian analysis of hazard rate, change point, and cost-optimal burn-in time for electronic devices. IEEE Transactions on Reliability, 59(1):132-138, 2010. 